\title{
PROTECCIÓN OSTEOARTICULAR DE Zea mays L. VARIEDAD MORADA (MAÍZ MORADO) EN ARTRITIS EXPERIMENTAL EN RATAS
}

\author{
Daisy Flores-Cortez 1,4,a , Eduardo Villalobos-Pacheco ${ }^{2,4, d}$, Armando Rojo-Mejia1,3,c, \\ Manuel Palomino-Yamamoto ${ }^{1,4, b}$, Yngerman Martin Ramirez $z^{5, e}$
}

\begin{abstract}
RESUMEN
Objetivos. Evaluar el efecto protector de Zea mays L. variedad morada (maíz morado) frente a la respuesta inflamatoria y daño osteoarticular en ratas con artritis experimental. Materiales y métodos. Se emplearon 65 ratas Holtzman, asignadas en siete grupos: $\mathrm{G} 1(n=5)$ : control, $\mathrm{G} 2(\mathrm{n}=10)$ : pristane $(\mathrm{PIA})+$ agua destilada, $\mathrm{G} 3(\mathrm{n}=10)$ : PIA + metotrexate

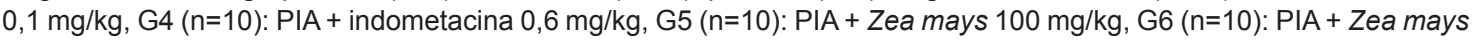
$1000 \mathrm{mg} / \mathrm{kg}$ y G7 (n=10): PIA + metotrexate $0,1 \mathrm{mg} / \mathrm{kg}+$ Zea mays $1000 \mathrm{mg} / \mathrm{kg}$. Los tratamientos fueron administrados mediante cánula orogástrica diariamente durante 21 días; el pristane se administró vía subdérmica solo el día 1 . Se registró el volumen de pata trasera con un pletismometro digital. El análisis radiológico de las patas se evaluó según los criterios de Clark modificado. Resultados. El porcentaje de inflamación al final del experimento fue: (G1) 1,50

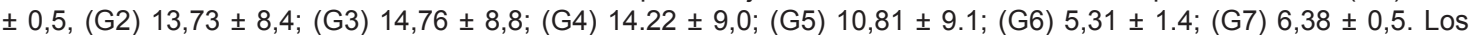
puntajes radiológicos de las áreas afectadas fueron: (G1) 0,6; (G2) 3,5; (G3) 0,6; (G4) 1,7; (G5) 1,9; (G6) 1,4; (G7) 1,0. Solo los grupos Zea mays L. $1000 \mathrm{mg} / \mathrm{kg}$ y metotrexate + Zea mays $L$. $1000 \mathrm{mg} / \mathrm{kg}$ mostraron una respuesta inflamatoria significativamente menor $(p<0,05)$ y mostraron puntajes articulares significativamente bajos en relación a PIA. Conclusiones. El Zea mays L. (maíz morado) reduce el proceso inflamatorio y las modificaciones radiológicas de la artritis inducida por PIA en ratas de modo dosis dependiente.
\end{abstract}

Palabras clave: Maíz morado; Plantas medicinales; Fitoterapia; Artritis reumatoide (Fuente: Decs BIREME).

\section{OSTEOARTICULAR PROTECTION OF Zea mays L. PURPLE VARIETY (PURPLE CORN) IN EXPERIMENTAL ARTHRITIS IN RATS}

\begin{abstract}
Objectives. To evaluate the protective effect of Zea mays L., purple variety (purple corn) against inflammatory response and osteoarticular damage in rats with experimental arthritis. Materials and Methods. Sixty-five Holtzman rats were used, assigned to seven groups: $G 1(n=5)$ : control; $G 2(n=10)$ : pristane (PIA) + distilled water; $G 3(n=10)$ : PIA + methotrexate $0.1 \mathrm{mg} / \mathrm{kg}$; G4 ( $\mathrm{n}=10)$ : PIA + indomethacin $0.6 \mathrm{mg} / \mathrm{kg} ; \mathrm{G} 5(\mathrm{n}=10)$ : PIA + Zea mays $100 \mathrm{mg} / \mathrm{kg} ; \mathrm{G} 6(\mathrm{n}=10)$ : $P I A+Z e a$ mays $1000 \mathrm{mg} / \mathrm{kg}$, and G7 ( $\mathrm{n}=10): P I A+$ methotrexate $0.1 \mathrm{mg} / \mathrm{kg}+$ Zea mays $1000 \mathrm{mg} / \mathrm{kg}$. Treatments were administered by orogastric cannula daily for 21 days; pristane was administered subdermal only on day 1 . Volume of hind leg was recorded with a digital plethysmometer. The radiological analysis of the legs was evaluated according to the modified Clark criteria. Results. The percentage of inflammation at the end of the experiment was: (G1) $1.50 \pm 0.5$ (G2) $13.73 \pm 8.4$; (G3) $14.76 \pm 8.8$; (G4) $14.22 \pm 9.0$; (G5) $10.81 \pm 9.1$; (G6) $5.31 \pm 1.4$; (G7) $6.38 \pm 0.5$. The radiological scores of the affected areas were: (G1) 0.6; (G2) 3.5; (G3) 0.6; (G4) 1.7; (G5) 1.9; (G6) 1.4; (G7) 1.0. Only the groups Zea mays $L$. $1000 \mathrm{mg} / \mathrm{kg}$ and methotrexate + Zea mays $L .1000 \mathrm{mg} / \mathrm{kg}$ showed a significantly lower inflammatory response $(p<0.05)$ and showed significantly lower joint scores in relation to PIA. Conclusions. Zea mays $L$. (purple corn) reduces the inflammatory process and radiological modifications of PIA-induced arthritis in rats in a dose-dependent manner.
\end{abstract}

Keywords: Purple corn; Medicinal plants; Phytotherapy; Rheumatoid arthritis (source: MeSH NLM).

\footnotetext{
Laboratorio de Farmacología. Facultad de Medicina Humana. Universidad Nacional Mayor de San Marcos. Lima, Perú. Sección de Fisiología. Facultad de Medicina Humana. Universidad Nacional Mayor de San Marcos. Lima, Perú.

Clínica Juan Pablo. Lima, Perú.

Centro de Investigación en Recursos Naturales (CIRNA). Lima, Perú.

Instituto Nacional de Salud del Niño. Lima, Perú.

Médico cirujano, doctor en Ciencias de la Salud; ${ }^{\mathrm{b}}$ médico dermatólogo, doctor en Medicina; ${ }^{\mathrm{c}}$ médico reumatólogo, doctor en Medicina; ${ }^{\mathrm{d}}$ licenciado en Obstetricia, magister en Fisiología; ${ }^{\mathrm{e}}$ licenciado en Radiología

Recibido: 06/02/2018 Aprobado: 04/07/2018 En línea: 28/09/2018
}

Citar como: Flores-Cortez D, Villalobos-Pacheco E, Rojo-Mejia A, Palomino-Yamamoto M, Martin Ramirez Y. Protección osteoarticular de Zea mays L. variedad morada (maíz morado) en artritis experimental en ratas. Rev Peru Med Exp Salud Publica. 2018;35(3):441-8. doi: 10.17843/rpmesp.2018.353.3454. 


\section{INTRODUCCIÓN}

La artritis reumatoide (AR) es una enfermedad inflamatoria sistémica de curso crónico, de causa multifactorial y origen autoinmune, cuya mayor característica es dolor y daño articular con progresiva discapacidad. En el 2004 la prevalencia de la enfermedad en Lima metropolitana fue de $0,32 \%{ }^{(1)}$; sin embargo, en el 2009 los resultados del primer estudio CopCORD en Perú señala una prevalencia de $0,51 \%{ }^{(2)}$. La AR puede llevar al deterioro de la calidad de vida del paciente, de su familia y de la sociedad en la que se desenvuelve, resultando en un alto costo directo e indirecto. En Perú, el costo de la medicación en los primeros dos años de la enfermedad representa el 55,6 \% ${ }^{(2)}$.

Las patologías articulares inflamatorias crónicas tienen en común un aumento de la respuesta inflamatoria del estrés oxidativo, lo que resulta en alteraciones histológicas progresivas y síntomas discapacitantes ${ }^{(3)}$. Los productos naturales, utilizados por milenios en la medicina tradicional, son una alternativa prometedora, de bajo costo, con menores eventos adversos y con eficacia comparable con la de los medicamentos convencionales en patologías articulares y que podría complementar el tratamiento convencional $(4,5)$. Sin embargo, en la mayoría de los casos hay escasez de evidencia clínica en cuanto a los niveles de seguridad, eficacia y calidad, a efecto de hacer llegar un producto terapéutico altamente confiable a la población.

El Zea mays L. (variedad morada) es un cereal que se ha cultivado en Perú desde épocas prehispánicas; su antigüedad está comprobada por las mazorcas encontradas en las tumbas, así como las representaciones en la cerámica precolombina. Su principal uso se da en la preparación de chicha y mazamorra morada; actualmente se preparan extractos acuosos atomizados para ser comercializados como colorantes naturales en Sudamérica y más recientemente en Asia y Europa ${ }^{(6)}$. Además del uso culinario, el maíz morado ha mostrado tener altos niveles de antocianinas, lo que le confiere el color morado al fruto. Asimismo, el maíz morado ha demostrado tener efecto protector significativo en diferentes modelos preclínicos de enfermedades crónicas como ateroesclerosis, hipertensión arterial, artritis y diabetes mellitus, básicamente debido al rol antioxidante de las antocianinas presentes en la especie ${ }^{(7-9)}$.

Mediante el modelo de artritis experimental en roedores inducido por pristane se induce una respuesta autoinmune específica, mediada por células T que afecta sobre todo a las uniones periféricas cartilaginosas y diartrodiales, produciendo cambios patológicos progresivos y crónicos que incluyen sinovitis con infiltración de polimorfonucleares y mononucleares, formación de panus, erosión de hueso y cartílago, y fibrosis articular. Este modelo comparte muchas características clínicas, histológicas, serológicas y genéticas de la artritis en humanos ${ }^{(10,11)}$, lo cual la hace atractiva para la evaluación de agentes terapéuticos potenciales para el manejo de esta enfermedad.

\section{MENSAJES CLAVE}

Motivación para realizar el estudio. Las patologías inflamatorias articulares son una condición clínica crónica de difícil control por lo que es necesario la búsqueda de alternativas que coadyuven al tratamiento. El maíz morado contiene antocianinas con propiedades terapéuticas antiinflamatorias. Sin embargo, no hay suficiente evidencia de su actividad en patologías articulares.

Principales hallazgos. El empleo oral de antocianinas derivados de Zea mays L. variedad morada (maíz morado) presentó actividad antinflamatoria, además de modificaciones radiológicas de la artritis inducida por pristane.

Implicancias. Promover la potencialidad terapéutica de Zea mays L. variedad morada (maíz morado) para su empleo como una alternativa coadyuvante y de fácil acceso en el tratamiento de patologías reumatológicas.

En el tratamiento de la AR se usan drogas antinflamatorias no esteroideas y fármacos que inducen remisión de la enfermedad. Entre estas drogas están la indometacina y el metotrexate, ambos usados como antinflamatorios ${ }^{(12,13)}$. Es reconocida la efectividad de estas drogas tanto experimental como clínicamente, por lo que se consideró de utilidad emplearlas como drogas comparativas con el producto de experimentación.

La artritis requiere tratamiento farmacológico a largo plazo, con medicamentos que tienen múltiples efectos adversos y esquemas complejos de dosificación, lo que contribuye a bajas cifras de adherencia a los esquemas terapéuticos recomendados ${ }^{(14)}$. Esto justifica la realización de estudios que busquen nuevas alternativas terapéuticas, como los recursos vegetales con potencial medicinal.

El objetivo del presente estudio fue evaluar el efecto protector osteoarticular del extracto de Zea mays $L$. variedad morada en la progresión de artritis experimental inducido en ratas.

\section{MATERIALES Y MÉTODOS}

\section{DISEÑO DEL ESTUDIO}

Se realizó un estudio experimental con dos grupos controles. Un grupo $(n=10)$ con la enfermedad inducida y un segundo grupo $(n=5)$ con animales sin la enfermedad. Para la asignación de las ratas a los grupos de tratamiento se empleó un muestreo aleatorio simple.

\section{ANIMALES}

Se emplearon 65 ratas de la cepa Holtzman, hembras de diez semanas, adquiridas en el Instituto Nacional de Salud (INS), las cuales fueron alojadas en el bioterio de la Facultad de Medicina de la Universidad Nacional 
Mayor de San Marcos, en jaulas metálicas con un ciclo de luz-oscuridad 12:12 h, a una temperatura ambiental aproximada de $23{ }^{\circ} \mathrm{C}$. Asimismo, recibieron una dieta balanceada peletizada y agua ad libitum. El tiempo de acondicionamiento previo al inicio del experimento fue de siete días.

\section{EXTRACCIÓN DE ANTOCIANINAS}

Se empleó el fruto de Zea mays $L$. variedad morada obtenido en la Provincia de La Oroya, Departamento de Junín e identificada por el área de botánica del Museo de Historia Natural. Para la obtención del extracto y de las antocianinas se empleó la técnica de Salinas et al. ${ }^{(15)}$ que consistió en la maceración de tres disolventes distintos: etanol, ácido acético y agua, en proporciones de 10:1:9, respectivamente. Cada litro de solución fue mezclado con 250 g de maíz morado seco y molido, y macerado durante siete días. El extracto filtrado fue evaporado y concentrado en una cámara de vacío a una presión menor de $50 \mathrm{mmHg}$ por un periodo mínimo de seis horas.

\section{PROCEDIMIENTO EXPERIMENTAL}

Se empleó el modelo de artritis experimental inducido por pristane $(\mathrm{PIA})^{(10)}$. Desde el día 1 hasta el día 21, todas las ratas $(n=60)$ fueron inmunizadas con 200 uL de PIAaplicado vía subdérmica en la base de la cola. Inmediatamente; los animales fueron distribuidos aleatoriamente y colocados en grupos, recibiendo tratamiento de la siguiente manera: G1 ( $n=5)$ : control (agua destilada $1 \mathrm{ml} / 100 \mathrm{~g}) ; \mathrm{G} 2(\mathrm{n}=10)$ : PIA + agua destilada; $\mathrm{G} 3(\mathrm{n}=10): \mathrm{PIA}+$ metotrexate $0,1 \mathrm{mg} / \mathrm{kg}$; G4 ( $\mathrm{n}=10): \mathrm{PIA}+$ indometacina $0,6 \mathrm{mg} / \mathrm{kg} ; \mathrm{G} 5(\mathrm{n}=10): \mathrm{PIA}$ + Zea mays L. $100 \mathrm{mg} / \mathrm{kg} ; \mathrm{G} 6(\mathrm{n}=10)$ : PIA+ Zea mays $L$. $1000 \mathrm{mg} / \mathrm{kg}$ y $\mathrm{G} 7(\mathrm{n}=10): \mathrm{PIA}+$ metotrexate $0,1 \mathrm{mg} / \mathrm{kg}+$ Zea mays L. $1000 \mathrm{mg} / \mathrm{kg}$. EI PIA fue administrado por única vez el primer día del experimento; mientras que los demás tratamientos fueron administrados mediante una cánula orogástrica diariamente, durante 21 días.

Se registró el volumen de la pata trasera, basal y semanal, hasta el término del experimento, mediante el empleo de un plestismometro digital (LE7500). El edema de la pata se expresó como el incremento de volumen de la pata por cada medición:

El día 22, las ratas fueron eutanizadas y las patas traseras

$$
\% \text { inflamación }=\frac{(\mathrm{Vtx}-\mathrm{Vt0})}{\mathrm{Vt} 0} \times 100
$$

Donde Vtx es el volumen de la pata inflamada a un tiempo $x$ y Vt0 es el volumen normal de la pata

seccionadas para su evaluación radiológica. Todas las radiografías fueron tomadas con film de rayos $X$ sensible al verde, estableciéndose técnicas radiográficas de $46 \mathrm{Kv}$, con $5 \mathrm{mAs}$ y $50 \mathrm{~mA}$ como foco fino. Las patas fueron colocadas $60 \mathrm{~cm}$ por debajo de la fuente de rayos $X$. Cada imagen radiológica fue evaluada ciegamente siguiendo los criterios de Clark modificado por Kawai ${ }^{(16)}$, tomando en cuenta los parámetros de desmineralización ósea, erosión ósea, periostitis y alineamiento de falanges con una escala de 0-3 (donde 0 indica normal y 3 indica cambios severos) para cada característica, tal como se describe en la tabla 1. La suma de estos cuatro hallazgos, definidos como puntuación articular, se obtuvo luego para cada pata trasera. Los puntajes articulares mínimo y máximo fueron 0 y 12 , respectivamente.

\section{ANÁLISIS ESTADÍSTICO}

La evaluación de la actividad protectora articular se realizó analizando dos parámetros: porcentaje de inflamación y puntuación radiológica de las patas traseras. Para la comparación de estos parámetros se tuvieron las siguientes consideraciones: se empleó la prueba de KolmogorovSmirnov (K-S) para evaluar la normalidad de las variables y se determinó la existencia de diferencias significativas entre los grupos de tratamiento empleando la prueba Kruskal-Wallis; además, se empleó la prueba U de MannWhitney para las comparaciones pares entre cada grupo y los controles. Se consideró significativo un valor de $p<0,05$.

\section{ASPECTOS ÉTICOS}

Se tomaron en cuenta las recomendaciones éticas para el manejo de animales de experimentación según el Instituto Nacional de Salud del Perú. El método de eutanasia empleado fue la inducción de pentobarbital $60 \mathrm{mg} / \mathrm{kg}$; considerado un método aceptable para roedores por el Report of the AVMA Panel on Eutanasia (1993) y el Euthanasia of Experimental Animals, de la Unión Europea (1995) ${ }^{(17)}$.

Tabla 1. Valoración radiológica de los parámetros osteoarticulares según el método de Clark modificado por Kawai (16)

\begin{tabular}{lcccc}
\hline \multirow{2}{*}{ Características } & \multicolumn{4}{c}{ Puntaje articular } \\
\cline { 2 - 5 } & $\mathbf{0}$ & $\mathbf{1}$ & $\mathbf{2}$ & $\mathbf{3}$ \\
\hline Desmineralización ósea & No presente & Poco significativa & Claramente significativa & Pronunciada \\
Erosión ósea y de cartílago & No presente & Poco significativa & Claramente significativa & Pronunciada \\
Periostitis & No presente & Poco significativa & Claramente significativa & Pronunciada \\
Alineamiento de falanges & Normal & Cambios leves & Cambios moderador & Cambios severos \\
\hline
\end{tabular}

Para cada parámetro, una puntuación de 0 indica normalidad y 3 indica cambios pronunciados o severos de la enfermedad. Los puntajes articulares totales mínimos y máximos son 0 y 12 , respectivamente 


\section{RESULTADOS}

El grupo tratado con Zea mays L. $100 \mathrm{mg} / \mathrm{kg}$ mostró un incremento lento de la respuesta inflamatoria de la pata hasta el día ocho; luego del cual mostró porcentajes de inflamación similares al grupo tratado con sólo PIA. El pico máximo de inflamación en el grupo Zea mays $L .1000 \mathrm{mg} / \mathrm{kg}$ fue en el día cinco, luego del cual los porcentajes disminuyeron, manteniéndose por debajo del grupo con sólo PIA hasta el día 19 de tratamiento. Finalmente, el grupo tratado con metotrexate + Zea mays L. $1000 \mathrm{mg} / \mathrm{kg}$, presentó un mayor porcentaje de inflamación en el día cinco, manteniéndose porcentajes de inflamación cercanos al grupo Zea mays L. $1000 \mathrm{mg} / \mathrm{kg}$. Las ratas tratadas con Zea mays $L .1000 \mathrm{mg} / \mathrm{kg}$ y metotrexate + Zea mays $L .1000 \mathrm{mg} / \mathrm{kg}$ mostraron una respuesta inflamatoria significativamente menor que los otros grupos de tratamiento $(p<0,05)$ (Figura 1).

Según el modelo estadístico de dos factores con medidas repetidas no se observaron diferencias significativas en el porcentaje de inflamación entre los grupos de tratamiento durante los primeros diez días de la enfermedad $(p<0,05)$. En el curso evolutivo de la inflamación crónica, durante todo el periodo de tratamiento, el grupo control presentó mediciones homogéneas de volumen de pata durante todo el periodo del experimento. Asimismo, los grupos tratados con pristane + Zea mays L. $1000 \mathrm{mg} / \mathrm{kg}$ y metotrexate + Zea mays $L .1000 \mathrm{mg} / \mathrm{kg}$ no presentaron cambios significativos en la respuesta inflamatoria inducida por PIA a lo largo del periodo experimental $(p<0,05)$ (Figura 1).

Los grupos tratados con PIA sólo y PIA + metotrexate presentaron un incremento de la respuesta inflamatoria a partir del día 15 de tratamiento. Antes, las diferencias no fueron significativas. Así mismo, los grupos tratados con PIA más indometacina y PIA + Zea mays $L .100 \mathrm{mg} / \mathrm{kg}$ mostraron una mayor respuesta inflamatoria a partir del día 12 de tratamiento (Figura 1).

Al final del tratamiento el porcentaje de inflamación acumulado es significativamente menor en el grupo Zea mays $L$. $1000 \mathrm{mg} / \mathrm{kg}$, seguido del grupo metotrexate + Zea mays $L$. 1000 mg/kg en relación al grupo de sólo PIA (Figura 2).

Se observó una respuesta antiinflamatoria en ratas artríticas tratadas con Zea mays L. $1000 \mathrm{mg} / \mathrm{kg}$ de $76,7 \%$, mientras que la misma respuesta fue $73,1 \%$ en el grupo tratado con metotrexate + Zea mays L. 1000 mg/kg (Figura 3).

Los animales tratados con Zea mays L. $1000 \mathrm{mg} / \mathrm{kg}$ y metotrexate + Zea mays $L .1000 \mathrm{mg} / \mathrm{kg}$ presentaron una

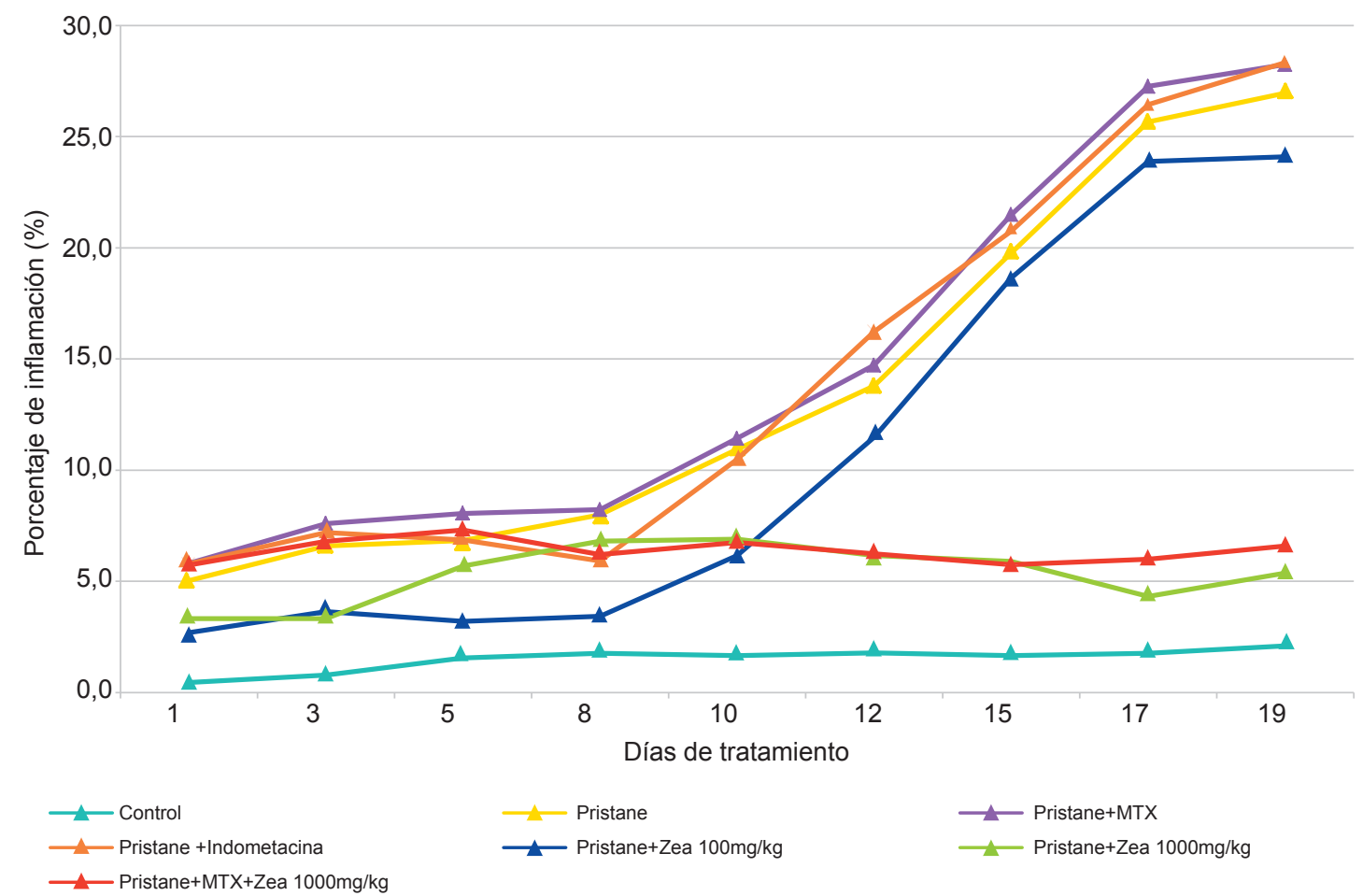

Control: ratas sanas sin pristane; MTX: metotrexate; Zea: extracto de Zea mays $L$.

Porcentaje de inflamación $=($ Volumen pata día $X-$ Volumen de pata día 1$) /$ Volumen de pata día 1 x 100

Figura 1. Porcentaje de inflamación en ratas artríticas inducida por pristane tratadas con Zea mays $L$. variedad morada 


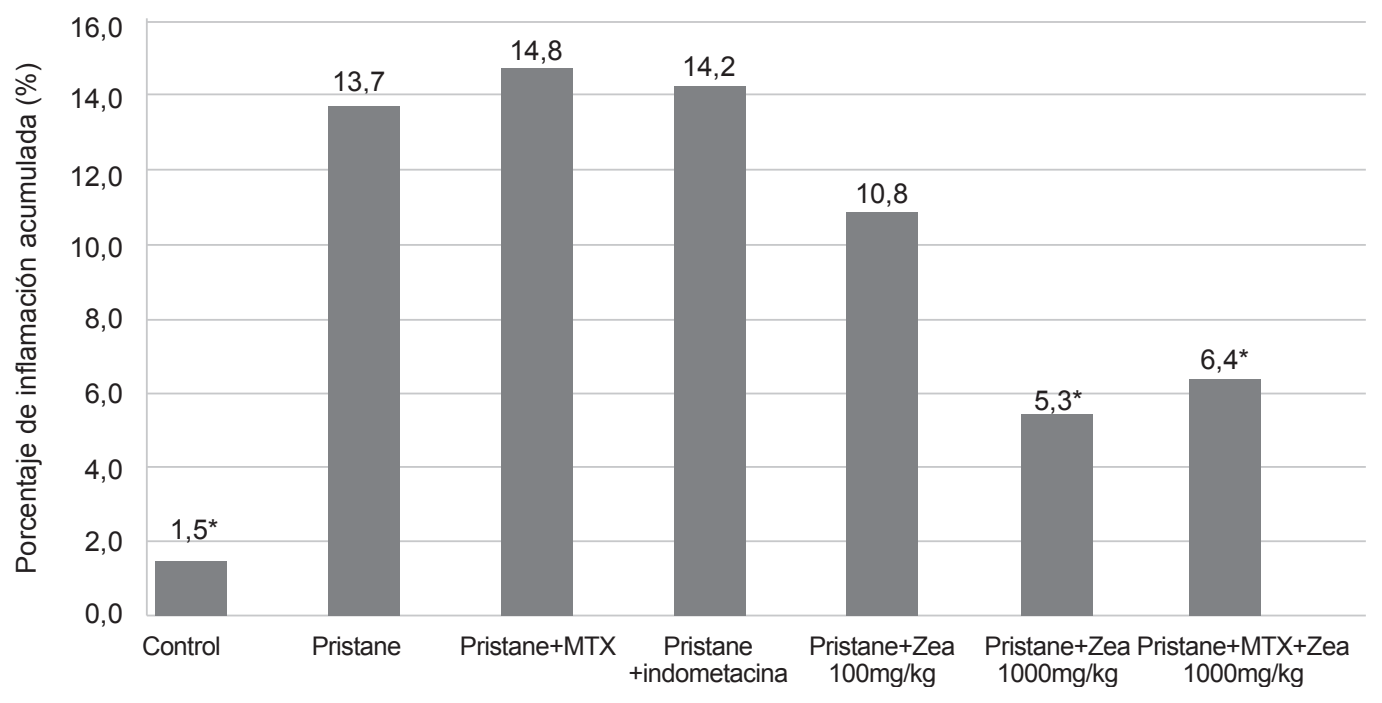

Control: ratas sanas sin pristane; MTX: metotrexate; Zea: extracto de Zea mays $L$.

Porcentaje de inflamación acumulada $(\%)=$ promedio de porcentaje de inflamación hasta el final del tratamiento

* Diferencia significativa $(p<0,05)$ en comparación con pristane, (prueba U Mann Whitney)

Figura 2. Porcentaje de inflamación acumulada al final del tratamiento en ratas con artritis tratadas con Zea mays $L$. variedad morada

puntuación articular total significativamente menor al grupo de sólo PIA (Tabla 2).

En el grupo tratado con PIA se evidencia erosión ósea y de cartílago significativamente mayor que los otros grupos de tratamiento. También se presentó periostitis en animales tratados con PIA, indometacina y Zea mays $L .100 \mathrm{mg} / \mathrm{kg}$. Las ratas tratadas con Zea mays $L .1000 \mathrm{mg} / \mathrm{kg}$ + metotrexate presentaron un menor grado de desmineralización ósea, periostitis, erosión ósea y de cartílago (Tabla 2) (Figura 4).

\section{DISCUSIÓN}

Los animales tratados con Zea mays L. $1000 \mathrm{mg} / \mathrm{kg}$ y metotrexate + Zea mays L. $1000 \mathrm{mg} / \mathrm{kg}$ presentaron una respuesta inflamatoria y daño articular significativamente menor en ratas con artritis experimental. El Zea mays $L$. $1000 \mathrm{mg} / \mathrm{kg}$ como monoterapia o con metotrexate presentó la misma eficacia antiinflamatoria $(5,3 \%$ vs $6,3 \%$ de inflamación acumulada). Asimismo, se observó que el Zea mays $L$. reduce la respuesta inflamatoria al inhibir el edema

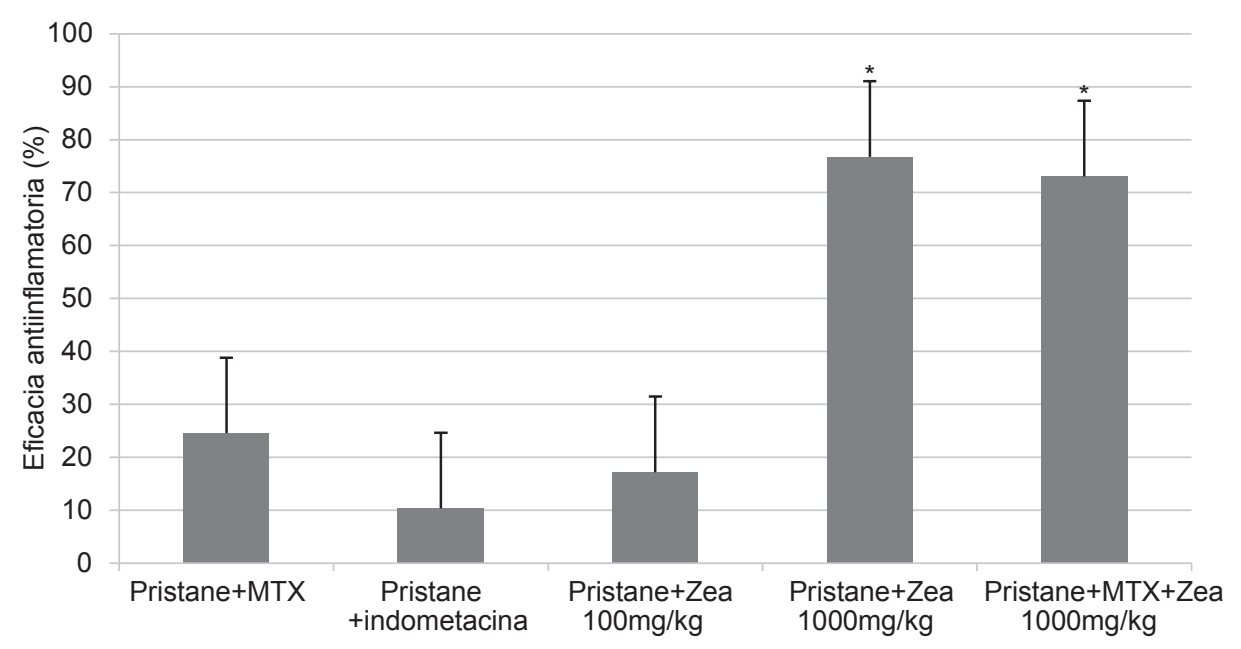

MTX: metotrexate; Zea: extracto de Zea mays $L$.

Eficacia antinflamatoria $=(100-$ porcentaje de inflamación $)$

*Diferencia significativa $(p<0,05)$ en comparación con pristane (prueba U Mann Whitney)

Figura 3. Eficacia antinflamatoria en ratas artríticas al finalizar el tratamiento con Zea mays $L$. variedad morada 
Tabla 2. Efecto de Zea mays $L$. variedad morada en la destrucción articular en la pata trasera de ratas con artritis inducida por pristane, según el método de Clark modificado por Kawai ${ }^{(16)}$

\begin{tabular}{|c|c|c|c|c|c|c|}
\hline \multirow[b]{2}{*}{ Tratamiento } & \multicolumn{5}{|c|}{ Puntaje articular } & \multirow[b]{2}{*}{ Valor de $p$} \\
\hline & $\begin{array}{c}\text { Desmineralización } \\
\text { ósea }\end{array}$ & $\begin{array}{l}\text { Erosión ósea } \\
\text { y de cartílago }\end{array}$ & Periostitis & $\begin{array}{l}\text { Alineamiento } \\
\text { de falanges }\end{array}$ & $\begin{array}{c}\text { Puntaje } \\
\text { total }\end{array}$ & \\
\hline Control & 0,2 & 0,4 & 0,0 & 0,0 & 0,6 & - \\
\hline Pristane & 0,7 & 1,4 & 0,7 & 0,7 & 3,5 & -- \\
\hline Pristane+MTX & 0,3 & 0,3 & 0,0 & 0,0 & 0,6 & $0,02^{*}$ \\
\hline Pristane +indometacina & 0,5 & 0,7 & 0,4 & 0,2 & 1,7 & $0,04^{*}$ \\
\hline Pristane+Zea $100 \mathrm{mg} / \mathrm{kg}$ & 0,4 & 0,7 & 0,3 & 0,5 & 1,9 & 0,12 \\
\hline Pristane+Zea $1000 \mathrm{mg} / \mathrm{kg}$ & 0,4 & 0,5 & 0,2 & 0,3 & 1,4 & $0,03^{*}$ \\
\hline $\begin{array}{l}\text { Pristane+MTX+Zea } \\
1000 \mathrm{mg} / \mathrm{kg}\end{array}$ & 0,1 & 0,4 & 0,2 & 0,3 & 1,0 & $0,01^{*}$ \\
\hline
\end{tabular}

Control: ratas sanas sin pristane; MTX: metotrexate; Zea: extracto de Zea mays $L$.

* Diferencia significativa $(p<0.05)$ comparación de puntaje total con respecto al pristane (prueba U Mann Whitney)

de la pata de ratas con artritis inducida por PIA de un modo dosis dependiente, siendo la mayor eficacia observada con una dosis de $1000 \mathrm{mg} / \mathrm{kg}$. Los resultados de la asociación de metotrexate y Zea mays L. $1000 \mathrm{mg} / \mathrm{kg}$ no demuestran un efecto sinérgico significativo respecto al grupo Zea mays L.1000 mg/kg sólo.

El Zea mays L. variedad morada (maíz morado) ha adquirido gran interés en el campo de la investigación en salud. Los estudios fitoquímicos realizados señalan que el $30,5 \%$ a $47,1 \%$ de los compuestos fenólicos presentes en el maíz morado lo constituyen las antocianinas. Las principales antocianinas del maíz morado son el cianidin3-glúcosido (C3G), pelargonidin-3-glúcosido, peonidin3-glúcosido, cianidin-3-maloilglúcosido, pelargonidin-3maloilglúcosido y peonidin-3-maloilglúcosido; además, del $36 \%$ al $54 \%$ de las antocianinas son aciladas ${ }^{(18)}$. Son antioxidantes naturales que poseen actividad farmacológica, biológica y terapéutica contra los radicales libres de oxígeno (ROS), el cual juega un rol importante en el daño tisular, a nivel articular y de otros tejidos con afecciones isquémicas, en tumores y en afecciones carcinogénicas ${ }^{(19)}$.

El C3G tiene actividad antioxidante significativa en ratas in vivo y como capturador de radicales superóxido e hidroxilo ${ }^{(20,21)}$. Así también, se ha demostrado que la antocianina cianidin-3-OB-D- glucósido reduce el edema en patas de ratas con artritis experimental y alivia la reacción inflamatoria en las articulaciones mediante inhibición de citoquinas proinflamatorias como TNFa, en un proceso inflamatorio agudo y un mecanismo antioxidante, a través del incremento de la actividad de glutatión reducido y de la superóxido dismutasa, que mejoran la capacidad antioxidante total y secuestran a los radicales libres.

Asimismo, la disminución de los niveles de prostaglandina E2 en los tejidos de las patas y del TNFa sérico; sugiere un posible mecanismo farmacológico en el control del proceso inflamatorio articular. Las proantocianinas también
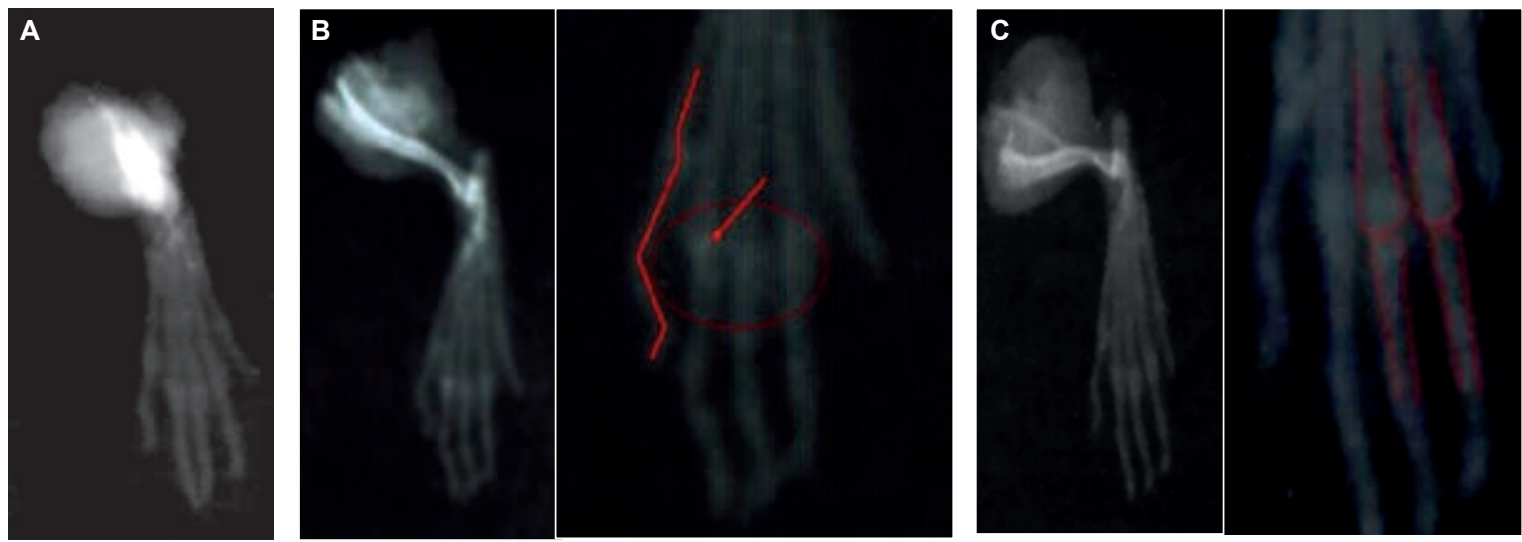

Figura 4. (A) Radiografía de pata normal, (B) Desalineamiento de falange del primer dedo de la pata derecha de ratas con artritis inducida por pristane, (C) Deformación articular de tercer y cuarta falange de la pata derecha de ratas con artritis inducida por pristane 
atenúan la expresión de las moléculas de adhesión (ICAM1, VCAM-1 y E-selectina) e incrementan la cantidad de formación de malondialdehido en el plasma de pacientes ${ }^{(22)}$. Karlsen et al. ${ }^{(23)}$ demuestran que las antocianinas inhiben la activación del factor nuclear kappa B en monocitos y reduce las concentraciones plasmáticas de mediadores proinflamatorios en sujetos sanos.

Nosotros planteamos que las antocianinas de Zea mays $L$. reducen la respuesta inflamatoria en la articulación maleolar, disminuyendo la progresión de la enfermedad, esto se ha demostrado con los resultados del análisis radiológico, donde se observa que el Zea mays $L$. reduce significativamente los parámetros radiológicos tales como: desmineralización y erosión ósea y del cartílago, periostitis y alineamiento de falanges, comparado con ratas tratadas con sólo PIA.

En la artritis, el estrés oxidativo induce inestabilidad genómica, senescencia replicativa y disfunción de condrocitos en el cartílago, sugiriendo que los ROS conducen a senescencia de condrocitos y envejecimiento del cartílago, y que podría ser responsable para el desarrollo de osteoartritis. Los nuevos esfuerzos para prevenir el desarrolloy progresión de osteoartritis puede incluir estrategias e intervenciones con el objetivo de reducir el daño oxidativo en el cartílago articular (24). Los cambios radiológicos en ratas con artritis inducida por PIA a similitud de los que ocurre en la enfermedad en los seres humanos, podría reforzar la validez del método empleado y por tanto, de los presentes resultados.

Los ROS son producidos en el sitio de la sinovitis por macrófagos y polimorfonucleares o por reperfusión mecánica y pueden contribuir al mantenimiento de la inflamación mediante la activación de moléculas inflamatorias que conducen a la destrucción del cartílago articular. Se ha observado que pacientes con artritis presentan un incremento de ROS y un incremento en el índice del estrés oxidativo, lo que se asocia a degeneración articular (25-27). Por tanto, los nuevos esfuerzos para prevenir el desarrollo y progresión de artritis deben incluir estrategias e intervenciones con el objetivo de reducir el daño oxidativo en el cartílago articular (24). Finalmente, las antocianinas del Zea mays $L$. variedad morada han demostrado un efecto antiproliferativo en carcinogénesis colorrectal ${ }^{(28)}$, interfiriendo el posible efecto inmunosupresor e inhibidor de la proliferación de panus sinovial ${ }^{(29)}$.

Como limitante podemos mencionar que, al ser un estudio experimental, nuestros resultados no necesariamente serán aplicables para el tratamiento de la artritis reumatoide en seres humanos; sin embargo, al igual que muchos otros estudios, consideramos que constituye un aporte a la investigación de este producto natural. Asimismo, por tratarse de una investigación sobre la eficacia de un producto vegetal en la evolución de la artritis experimental inducida por PIA, se sugiere la realización de estudios con metodologías más rigurosas que incluyan marcadores bioquímicos e inmunológicos de la inflamación.

Sería pertinente también, purificar la técnica de aislamiento de antocianina del maíz morado, extraer los metabolitos activos y encontrar la dosis efectiva máxima y tóxica. La mayor estabilidad y capacidad antioxidante de las antocianinas del maíz morado frente a otras especies vegetales ${ }^{(30)}$, sumado al aporte científico que presentamos en nuestros resultados, abre la posibilidad de revalorar este producto natural originario del Perú en el campo de la medicina y en especial en reumatología, considerando la posibilidad de ser empleado, en el futuro, como terapia adyuvante de la artritis reumatoide.

En conclusión, en este estudio, se ha determinado una tendencia a la mejoría en el proceso inflamatorio articular en la pata de rata con artritis inducida por pristane. Sobre todo, en los grupos tratados con las mayores dosis de Zea Mays $L$. variedad morada, así como en los grupos asociados con metotrexate observando, además, una menor progresión radiológica.

Contribuciones de autoría: DFC, EVP, MPY y ARM han participado en la concepción, desarrollo de la investigación y redacción del artículo. Además, DFC, EVP, MPY y ARM realizaron la revisión crítica del artículo. YMR realizó el análisis e interpretación de las imágenes radiológicas. Todos los autores participaron en la evaluación de resultados, su interpretación y la aprobación de la versión final del artículo.

Fuentes de financiamiento: El proyecto fue financiado por el Consejo Nacional de Ciencia y Tecnología (CONCYTEC).

Conflicto de interés: Los autores declaran no tener conflictos de interés.

\section{REFERENCIAS BIBLIOGRÁFICAS}

1. Medina M, Acevedo E, Gutierrez C, Perich R, Sanchez-Torres A, De Leon $\mathrm{DP}$, et al. Low prevalence of rheumatoid arthritis in an urban mestizo population. JCR J Clin Rheumatol. 2006;12(4):S5.

2. Gamboa DR, Medina M, Acevedo E, Pastor C, Cucho J, Gutiérrez C, et al. Prevalencia de enfermedades reumatologicas y discapacidad en una comunidad urbano-marginal: resultados del primer estudio CoPCord en el Perú. Rev Per Reum. 2009;15(1):40-6.

3. Suantawee T, Tantavisut S, Adisakwattana S, Tanavalee A, Yuktanandana P, Anomasiri W, Deepaisarnsakul
B HS. Oxidative Stress, Vitamin E, and Antioxidant Capacity in Knee Osteoarthritis. J Clin Diagn Res. 2013;7(9):1855-9.

4. Dragos D, Gilca M, Gaman L, Vlad A, Iosif L, Stoian I, et al. Phytomedicine in Joint Disorders. Nutrients. 2017;9(1):70. 
5. Elisha IL, Dzoyem J-P, McGaw LJ, Botha FS, Eloff JN. The anti-arthritic, antiinflammatory, antioxidant activity and relationships with total phenolics and total flavonoids of nine South African plants used traditionally to treat arthritis. BMC Complement Altern Med. 2016;16(1):307.

6. Biocomercio-Perú/PROMPEX [Internet]. Lima, Peru. Evolución de la producción de antocianina 20002002 (Perú). 2004. [Citado el 5 de abril de 2018]. Disponible en: http:// www.monografias.com/trabajos58/ produccion-antocianina/produccionantocianina3.shtml

7. HuangB, WangZ, ParkJH, Ryu OH, Choi MK, Lee J-Y, et al. Anti-diabetic effect of purple corn extract on C57BL/KsJ db/db mice. Nutr Res Pract. 2015;9(1):22-9.

8. Li J, Lim SS, Lee J-Y, Kim J-K, Kang S-W, Kim J-L, et al. Purple corn anthocyanins dampened high-glucose-induced mesangial fibrosis and inflammation: possible renoprotective role in diabetic nephropathy. J Nutr Biochem. 2012;23(4):320-31.

9. Flores D. Efecto del extracto acuoso de Zea mays L variedad morada sobre la presión arterial en ratas [Tesis Maestria]. Lima: Facultad de Medicina, Universidad Nacional Mayor de San Marcos; 2003.

10. Tuncel J, Haag S, Hoffmann $M H$, Yau ACY, Hultqvist M, Olofsson P, et al. Animal Models of Rheumatoid Arthritis (I): Pristane-Induced Arthritis in the Rat. Zhou H, editor. PLoS One. 2016;11(5):e0155936.

11. Hoffmann $\mathrm{MH}$, Hopf R, Niederreiter B, Redl H, Smolen JS, Steiner G. Gait changes precede overt arthritis and strongly correlate with symptoms and histopathological events in pristaneinduced arthritis. Arthritis Res Ther. 2010;12(2):R41.

12. Masferrer JL, Zweifel BS, Manning PT, Hauser SD, Leahy KM, Smith WG, et al. Selective inhibition of inducible cyclooxygenase 2 in vivo is antiinflammatory and nonulcerogenic. Proc Natl Acad Sci U S A. 1994;91(8):3228-32.
13. Cronstein BN. Low-Dose Methotrexate: A Mainstay in the Treatment of Rheumatoid Arthritis. Pharmacol Rev. 2005;57(2):163-72.

14. Ruiz C, Parada A, Urrego A, Gallego D. Adherencia al tratamiento en artritis reumatoide: condición indispensable para el control de la enfermedad. Rev Cuba Med Gen Integr. 2016;32(3).

15. Salinas, Y; Rubio, D; Diaz A. Extracción y uso de pigmentos del grano del maíz (Zea mays L) como colorantes en yogurt. ALAN. 2005;55(3):293-8.

16. Kawai S, Nagai K, Nishida S, Sakyo K, Murai E, Mizushima Y. Low-dose pulse methotrexate inhibits articular destruction of adjuvant arthritis in rats. J Pharm Pharmacol. 1997;49(2):213-5.

17. Close B, Banister K, Baumans V, Bernoth E-M, Bromage N, Bunyan J, et al. Recomendaciones para la Eutanasia de los Animales de Experimentación. Lab Anim (NY). 1997;31:1-32.

18. Salgado J, Menguan E. Determinación de compuestos fenólicos en estilos, estigmas, estambres y bracteas en la especie vegetal Zea mays L variedad morada [Tesis Pregrado]. Lima: Facultad de Farmacia y Bioquimica, Universidad Nacional Mayor de San Marcos; 2000.

19. Pedreschi R, Cisneros-Zevallos L. Antimutagenic and antioxidant properties of phenolic fractions from Andean purple corn (Zea mays L.). J Agric Food Chem. 2006 28;54(13):4557-67.

20. Tsuda T, Kato Y, Osawa T. Mechanism for the peroxynitrite scavenging activity by anthocyanins. FEBS Lett. 2000;484(3):207-10.

21. Kong J-M, Chia L-S, Goh N-K, Chia T-F, Brouillard R. Analysis and biological activities of anthocyanins. Phytochemistry. 2003;64(5):923-33.

22. Kalin R, Righi A, Del Rosso A, Bagchi D, Generini S, Cerinic MM, et al. Activin, a grape seed-derived proanthocyanidin extract, reduces plasma levels of oxidative stress and adhesion molecules (ICAM-1, VCAM-1 and E-selectin) in systemic sclerosis. Free Radic Res. 2002;36(8):819-25.
23. Karlsen A, Retterstøl L, Laake P, Paur I, Bøhn SK, Sandvik L, et al. Anthocyanins inhibit nuclear factor-kappaB activation in monocytes and reduce plasma concentrations of pro-inflammatory mediators in healthy adults. J Nutr. 2007;137(8):1951-4.

24. Yudoh $\mathrm{K}$, van Trieu N, Nakamura $\mathrm{H}$, Hongo-Masuko K, Kato T, Nishioka K. Potential involvement of oxidative stress in cartilage senescence and development of osteoarthritis: oxidative stress induces chondrocyte telomere instability and downregulation of chondrocyte function. Arthritis Res Ther. 2005;7(2):R380.

25. Altindag O, Karakoc M, Kocyigit A, Celik $\mathrm{H}$, Soran N. Increased DNA damage and oxidative stress in patients with rheumatoid arthritis. Clin Biochem. 2007;40(34):167-71.

26. Jaswal S, Mehta HC, Sood AK, Kaur J. Antioxidant status in rheumatoid arthritis and role of antioxidant therapy. Clin Chim Acta. 2003;338(1-2):123-9.

27. Yamada K, Nakamura T, Utsumi H. Enhanced intraarticular free radical reactions in adjuvant arthritis rats. Free Radic Res. 2006;40(5):455-60.

28. Hagiwara A, Miyashita K, Nakanishi T, Sano M, Tamano S, Kadota T, et al. Pronounced inhibition by a natural anthocyanin, purple corn color, of 2-amino-1-methyl-6-phenylimidazo[4,5-b] pyridine (PhIP)-associated colorectal carcinogenesis in male F344 rats pretreated with 1,2-dimethylhydrazine. Cancer Lett. 2001;171(1):17-25.

29. Hamilton JA. Hypothesis: in vitro evidence for the invasive and tumor-like properties of the rheumatoid pannus. J Rheumatol. 1983;10(6):845-51.

30. Gorriti A, Quispe F, Arroyo A JL, Córdova A, Jurado B, Santiago I, et al. Extracción de antocianinas de las corontas de Zea mays L. "Maiz morado." Cienc Invest. 2009;12(2):64-74.

Correspondencia: Daisy Flores Cortez

Dirección: Av. Grau 755. Lima 01, Perú

Teléfono: 016197000 anexo 4622

Correoelectrónico:dfloresc@unmsm.edu.pe 